\title{
Crescimento de prematuros de extremo baixo peso nos primeiros dois anos de vida
}

\author{
Growth of extremely low birth weight infants during the first two years of life
}

Ligia Maria S. S. Rugolo ${ }^{1}$, Maria Regina Bentlin², Antônio Rugolo Junior², Ivete Dalben³, Cleide Enoir P. Trindade ${ }^{4}$

\section{RESUMO}

Objetivo: Analisar o padrão de crescimento de prematuros de extremo baixo peso (EBP) até 24 meses de idade corrigida, a influência da displasia broncopulmonar (DBP) e os fatores de risco para falha de crescimento.

Métodos: Coorte de prematuros <1.000g de gestação única, nascidos e acompanhados em um centro terciário. $\mathrm{O}$ crescimento foi avaliado por meio de escores-z para peso, comprimento e perímetro cefálico ao nascimento, com 40 semanas, aos 3, 6, 12, 18 e 24 meses de idade corrigida. Dentre 81 sobreviventes, 70 foram estudados e estratificados em dois grupos: DBP $(n=41)$ e sem DBP $(n=29)$. Foi realizada análise bivariada com teste $t$ ou Mann-Whitney, qui-quadrado ou Exato de Fisher, e análise multivariada com regressão logística.

Resultados: Em ambos os grupos, o escore-z de peso diminuiu significantemente entre o nascimento e 40 semanas. Houve um pico de incremento nos escores-z de peso, comprimento e perímetro cefálico entre 40 semanas e três meses. No grupo sem DBP, os escores-z atingiram a faixa normal a partir dos seis meses e assim permaneceram até 24 meses de idade corrigida. Crianças com DBP tiveram menores escores-z de peso e perímetro cefálico no primeiro ano, mas equipararam-se às sem DBP no segundo ano de vida. A regressão logística mostrou que catch-down no escore-z de peso com 40 semanas foi fator de risco para falha de crescimento.

Conclusões: Prematuros EBP apresentam $c a t c h-u p$ precoce do crescimento nos primeiros dois anos. Crianças com DBP têm pior crescimento ponderal. A restrição do crescimento pós-natal prediz a falha de crescimento nos primeiros anos.

Palavras-chave: prematuro; recém-nascido de extremo baixo peso; displasia broncopulmonar; crescimento.

\section{ABSTRACT}

Objective: To evaluate the growth pattern of extremely low birth weight infants (ELBW) from birth to 24 months of adjusted gestational age (AA), the influence of bronchopulmonary dysplasia (BPD) and risk factors associated to growth failure.

Methods: This cohort study included all singleton inborn infants with birthweight $<1,000 \mathrm{~g}$, admitted in the followup clinic of a level III Perinatal Center. Weight, length and head circumference were measured at birth, 40 weeks, and 3, 6, 9, 12, 18, 24 months AA, and Z-scores were calculated. Out of the 82 survivors, 70 were studied and classified in two groups: BPD $(n=41)$ and no-BPD $(n=29)$. Statistical analysis included $t$-test or Mann-Withney, chi-square or Fisher Exact test, and multivariate logistic regression.

Results: In both groups, weight z-score decreased significantly between birth and 40 weeks AA. A peak incremental change in weight, length and head circumference z-scores occurred between 40 weeks and three months. Z-scores for the no-BPD group were close to the expected values by the age of six months and remained at these levels at 24 months AA. Children with BPD had lower z-scores for weight and head circumference in the first year of life, but no difference was found between BPD and no-BPD children in the second year of life. Regression analysis showed that catch-down in weight z-score at 40 weeks was a risk factor for failure to thrive.

Conclusions: ELBW infants experienced early catch-up growth during the first two years of life. ELBW with BPD had poor weight gain. Post-natal growth restriction predicts failure to thrive in infancy.

Key-words: infant, premature; infant, extremely low birth weight; bronchopulmonary dysplasia; growth.
${ }^{1}$ Professor livre-docente do Departamento de Pediatria da Faculdade de Medicina de Botucatu da Universidade Estadual Paulista (Unesp)

${ }^{2}$ Professor Assistente doutor do Departamento de Pediatria da Faculdade de Medicina de Botucatu da Unesp

3Professor assistente doutor do Departamento de Saúde Pública da Faculdade de Medicina de Botucatu da Unesp

${ }^{4}$ Professor titular do Departamento de Pediatria da Faculdade de Medicina de Botucatu da Unesp
Endereço para correspondência:

Ligia M. S. Souza Rugolo

Rua Dr. Cardoso de Almeida, 1.000, apto. 62

CEP 18.600-005 - Botucatu/SP

E-mail: ligiasr@fmb.unesp.br

Recebido em: 21/11/2006

Aprovado em: 26/2/2007 


\section{Introdução}

O aumento na sobrevida de prematuros cada vez menores tem gerado crescente preocupação quanto ao prognóstico de crescimento e desenvolvimento dessas crianças ${ }^{(1,2)}$. No seguimento ambulatorial, as questões mais freqüentemente formuladas pelas mães de recém-nascidos prematuros, as quais constituem motivo de preocupação para os familiares e os médicos, incluem: o prematuro será sempre pequeno? Quando irá recuperar seu crescimento e equiparar-se às crianças normais?

Prematuros de extremo baixo peso (EBP) são expostos a vários fatores que podem comprometer seu crescimento, destacando-se a limitação na oferta nutricional durante a internação em UTI, a inadequação nutricional após a alta, as doenças crônicas, a elevada morbidade e necessidade de reinternações nos primeiros anos, bem como a baixa condição socioeconômica familiar e a má qualidade dos cuidados no $\operatorname{lar}^{(1,3-6)}$. Dentre os prematuros de risco para problemas no crescimento, destacam-se aqueles com displasia broncopulmonar (DBP), nos quais se concentram muitos fatores comprometedores do crescimento, incluindo: trabalho respiratório aumentado, episódios de hipoxemia, necessidade de restrição hídrica e uso de corticóide pós-natal ${ }^{(2,7-9)}$.

Este estudo foi proposto para analisar o padrão de crescimento de prematuros de EBP e avaliar a repercussão da DBP no crescimento, durante os primeiros dois anos de vida. Procurou-se determinar quando e como ocorre o catch-up nos prematuros com e sem displasia, a freqüência de falha no crescimento e os fatores de risco associados.

\section{Métodos}

Trata-se de estudo unicêntrico de coorte, com prematuros de EBP, egressos de unidade de terapia intensiva (UTI) neonatal e acompanhados no Ambulatório de Seguimento de Recém-Nascidos de Alto Risco. O estudo foi aprovado pelo Comitê de Ética em Pesquisa da Faculdade de Medicina de Botucatu, Unesp.

Foram selecionados todos os prematuros $<1.000 \mathrm{~g}$, nascidos na maternidade do serviço no período de janeiro de 1990 a dezembro de 2000 e que tiveram alta hospitalar, o que correspondeu a 81 sobreviventes, dentre os 231 nascidos.

Para os 81 prematuros selecionados, foram levantadas as fichas do ambulatório e os prontuários, a partir dos quais os dados do presente estudo foram obtidos de forma retrospectiva. Foram incluídos no estudo os pacientes que preencheram os seguintes critérios: gestação única; alta hospitalar antes do sexto mês de vida; ausência de anomalias congênitas múltiplas, síndromes genéticas e infecções congênitas. Conforme estes critérios, quatro pacientes não foram incluídos. Foram excluído os pacientes seguidos por tempo inferior a 12 meses de idade corrigida e os que tiveram menos de três consultas no primeiro ano e duas no segundo ano de vida.

As seguintes definições foram adotadas no estudo:

- DBP: dependência de oxigênio aos 28 dias de vida, acompanhada de sinais clínicos e radiológicos de comprometimento pulmonar ${ }^{(10)}$;

- Catch-up ou crescimento acelerado: aumento 0,67 entre dois escores-z consecutivos ${ }^{(11)}$;

- Catch-down ou desaceleração do crescimento: diminuição 0,67 entre dois escores-z consecutivos ${ }^{(11)}$;

- Falha de crescimento: peso abaixo do percentil 5 na curva do CDC-NCHS 2000 $0^{(12)}$;

- Idade corrigida (IC): idade cronológica (em semanas) (40 - idade gestacional). Utilizada nos primeiros dois anos de vida ${ }^{(2)}$.

As medidas antropométricas foram obtidas em cada consulta pela equipe médica e de enfermagem fixa no setor e previamente treinada. Os pacientes foram pesados despidos, em balança infantil Filizzola ${ }^{\circledR}$, com precisão de $5 \mathrm{~g}$. O comprimento foi medido em decúbito dorsal, com estadiômetro de madeira. $\mathrm{O}$ perímetro cefálico foi medido com fita inelástica.

As medidas antropométricas do nascimento, com 40 semanas (termo), aos 3, 6, 9, 12, 18 e 24 meses de idade corrigida (IC) foram avaliadas pelo cálculo do escore-z, que indica o número de desvios padrão no qual a medida antropométrica da criança se distancia da média populacional, tendo como padrão de referência as curvas de crescimento do CDC-NCHS $2000^{(12)}$. Para o cálculo dos escores-z, utilizou-se o programa estatístico EPI-Info 2000. Inicialmente foram calculados os escores-z das medidas ao nascer e, a seguir, procedeu-se a correção da idade em função da prematuridade, considerando-se as medidas antropométricas com 40 semanas como aquelas correspondentes à data prevista para o nascimento.

Devido à variabilidade nas idades das crianças em cada consulta, a avaliação do crescimento foi efetuada por trimestres no primeiro ano e por semestres no segundo ano. Diante de mais de uma consulta no período, foi efetuada a média dos escores-z.

Foram avaliadas as variáveis:

- Sociodemográficas: procedência; situação conjugal; escolaridade em anos e ocupação da mãe e do pai, classificada conforme a Escala de Prestígio Ocupacional ${ }^{(13)}$;

- Gestacionais: consultas de pré-natal; tabagismo; doenças prévias e/ou na gestação, uso de corticóide antenatal e tipo de parto; 
- Neonatais: idade gestacional (estimada pela data precisa da última menstruação materna ou pelo ultra-som obstétrico antes de 20 semanas de gestação); peso de nascimento e sua adequação para a idade gestacional ${ }^{(14)}$; gênero; morbidade (presença de síndrome do desconforto respiratório - SDR, persistência do canal arterial - PCA com repercussão hemodinâmica, sepse clínica ou confirmada por culturas, meningite, doença metabólica óssea, enterocolite necrosante graus 2 e 3 , hemorragia periintraventricular graus 3 e $4^{(15)}$, retinopatia da prematuridade grau 2; uso de corticóide pós-natal e tempo de internação;

- Alimentação no primeiro ano: tempo de aleitamento materno exclusivo, aleitamento misto e artificial;

- Morbidade pós-alta: intercorrências que necessitaram de consulta médica e tratamento específico; internações.

Conforme a presença ou ausência de DBP, foram constituídos dois grupos de estudo: DBP e sem DBP. O desfecho de interesse foi o crescimento, caracterizado como ocorrência de "catch-up" no primeiro e segundo ano e/ou "falha de crescimento" aos 12 e 24 meses de idade corrigida.

Os dados foram analisados usando o programa SPSS, versão 8.0. Foi realizada estatística descritiva com o cálculo da distribuição de freqüências, médias e desvios padrão, medianas e percentis. As diferenças entre os grupos foram analisadas pelos testes: $t$ ou Mann-Whitney, qui-quadrado ou Exato de Fisher. Para o estudo dos prováveis fatores pré-natais, perinatais e neonatais preditores de falha no crescimento, utilizou-se a regressão logística com a estratégia stepwise selection. O nível de significância foi de 5\%.

\section{Resultados}

De acordo com os critérios propostos, 77 prematuros de EBP foram inicialmente incluídos e, destes, sete foram excluídos. A coorte foi então constituída com 70 prematuros, dos quais quatro evoluíram com hidrocefalia e foram submetidos à derivação ventrículo-peritoneal, motivo pelo qual não foram considerados na avaliação do perímetro cefálico.

A perda da coorte foi de 14\% entre 12-18 meses (cinco crianças com DBP e cinco sem DBP) e de outros $14 \%$ entre 18-24 meses (seis DBP e quatro sem DBP). Foram documentados três óbitos no segundo ano de vida. Nos demais casos, não foi possível identificar o motivo da evasão.

A caracterização sociodemográfica mostrou: $36 \%$ das famílias residentes no município, união estável em $77 \%$, menos que oito anos de estudo em $71 \%$ das mães e em $84 \%$ dos pais, predomínio de ocupação não remunerada entre as mães (70\%) e ocupações manuais especializadas ou não entre os pais $(74 \%)$. O acompanhamento pré-natal foi freqüente e $71 \%$ das gestantes tiveram intercorrências gestacionais. Um terço das mães era fumante e $44 \%$ receberam corticosteróide antenatal. O sofrimento fetal foi detectado em $41 \%$ das gestações e o parto cesáreo ocorreu em $60 \%$.

Os recém-nascidos com DBP corresponderam a 59\% da amostra, tiveram menor idade gestacional, comprimento e perímetro cefálico, apresentaram maior morbidade neonatal e tempo de internação mais prolongado, em comparação aos sem DBP (Tabela 1). A sepse ocorreu em $50 \%$ da amostra e a enterocolite necrosante em $16 \%$, sem diferença entre os grupos. Todos os prematuros receberam nutrição parenteral,

Tabela 1 - Características neonatais dos prematuros de extremo baixo peso, com e sem DBP

\begin{tabular}{lcccc}
\hline & $\begin{array}{c}\text { Total } \\
(\mathbf{n = 7 0})\end{array}$ & $\begin{array}{c}\text { Sem DBP } \\
(\mathbf{n = 2 9})\end{array}$ & $\begin{array}{c}\text { DBP } \\
(\mathbf{n = 4 1 )}\end{array}$ & Valor de $\boldsymbol{p}$ \\
\hline Idade gestacional (sem) & $28 \pm 1,9$ & $29 \pm 2,0$ & $27 \pm 1,7$ & 0,002 \\
Peso nascimento (g) & $853 \pm 107$ & $891 \pm 57$ & $828 \pm 126$ & 0,109 \\
Comprimento (cm) & $34,6 \pm 1,7$ & $35,2 \pm 1,4$ & $34,2 \pm 1,9$ & 0,017 \\
Perímetro cefálico (cm) & $24,8 \pm 1,2$ & $25,2 \pm 1,1$ & $24,5 \pm 1,2$ & 0,023 \\
Gênero feminino (\%) & 67 & 79 & 59 & 0,118 \\
Pequeno para a idade gestacional (\%) & 44 & 52 & 38 & 0,418 \\
Síndrome Desconforto Respiratório (\%) & 69 & 52 & 80 & 0,022 \\
Corticóide pós-natal (\%) & 34 & 4 & 56 & $<0,001$ \\
PCA com repercussão (\%) & 57 & 34 & 73 & 0,003 \\
Doença metabólica óssea (\%) & 51 & 28 & 66 & 0,004 \\
Hemorragia ventricular graus 3-4 (\%) & 14 & 7 & 19 & 0,178 \\
Retinopatia prematuridade graus 2-3 (\%) & 16 & 4 & 24 & 0,021 \\
Dias de internação (mediana) & 68 & 56 & 74 & $<0,001$ \\
\hline
\end{tabular}


e a alimentação via gástrica foi iniciada após a primeira semana, na maioria deles. Os prematuros, em média, tiveram alta com 37 semanas de idade corrigida, em aleitamento predominantemente misto. $\mathrm{O}$ aleitamento materno exclusivo ocorreu em apenas dois dos 70 prematuros. O desmame foi muito precoce: com 40 semanas, somente $40 \%$ recebiam aleitamento misto e, no final do primeiro trimestre, $21 \%$. No segundo trimestre, $95 \%$ estavam em aleitamento artificial.

A morbidade pós-alta foi elevada até os 18 meses e representada basicamente pelas infecções respiratórias. Os grupos só diferiram no segundo trimestre, quando os problemas respiratórios predominaram no grupo DBP $(53 \%$ versus $19 \% ; p=0,013)$. No primeiro ano de vida, $32 \%$ dos lactentes displásicos e $24 \%$ dos sem displasia foram hospitalizados $(p=0,673)$.

Os valores médios dos escores-z de peso, comprimento e perímetro cefálico estão apresentados na Figura 1 e nas Tabelas 2 a 4 . Nos dois grupos, ocorreu catch-down do peso entre o nascimento e 40 semanas, seguido por $c$ atch-up no primeiro trimestre. Nos displásicos, o catch-down inicial foi mais acentuado e a evolução ponderal foi pior no primeiro semestre, em comparação ao grupo sem DBP (Tabela 2 e Figura 1).

Os escores-z de comprimento foram substancialmente baixos ao nascimento, porém mostraram evolução mais satisfatória que os escores relativos ao peso, atingindo a faixa de normalidade ( -2 a zero) antes do final do primeiro ano, nos dois grupos. Só houve diferença entre os grupos com 40 semanas (Tabela 3 e Figura 1).

Nos dois grupos, os escores-z de perímetro cefálico foram muito baixos ao nascimento, com acentuado catch-up até três meses de idade corrigida, atingindo a faixa de normalidade (-2 a zero) no primeiro ano. Os displásicos tiveram perímetro cefálico significantemente menor entre 6-12 meses de idade corrigida (Tabela 4 e Figura 1).

A Tabela 5 mostra o número e o percentual de prematuros com e sem DBP que apresentaram catch-up aos 12 e 24 meses de IC. Para o peso, o percentual de crianças em fase de catchup aos 12 meses foi baixo e aumentou significantemente até 24 meses de idade corrigida, sem diferença entre os grupos. Para o comprimento e o perímetro cefálico, a ocorrência de catch-up aos 12 meses foi maior do que para o peso, e não houve diferença significativa entre os grupos e os momentos de avaliação.

Apesar da ocorrência de catch-up nos dois grupos de prematuros, elevada proporção deles não atingiu o percentil 5 da curva do NCHS até o final do segundo ano de vida, sendo tal percentual de $56 \%$ para o peso, $28 \%$ para o comprimento e $32 \%$ para o perímetro cefálico. A Figura 2 apresenta o per-

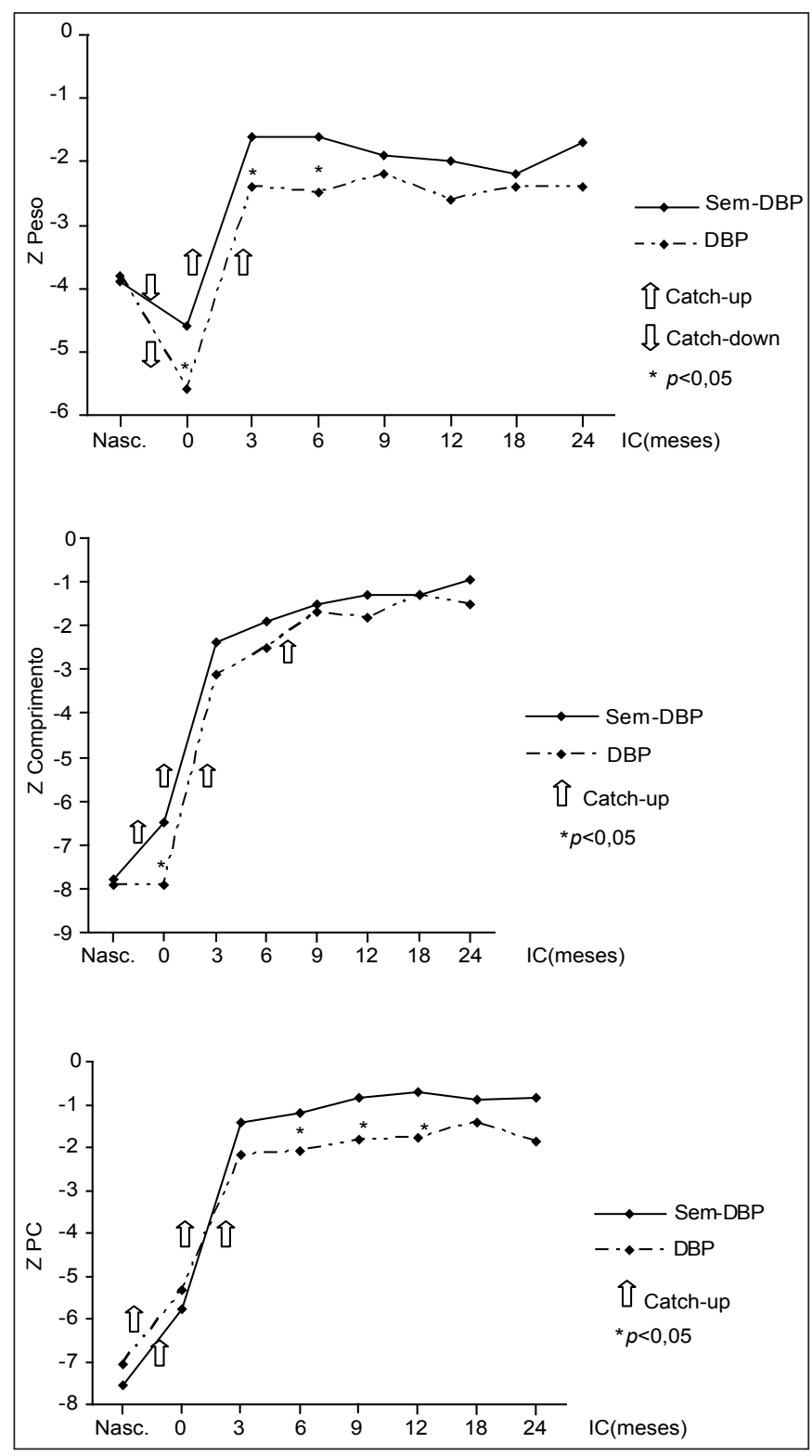

Figura 1 - Médias dos escores- $Z$ de peso, comprimento e perímetro cefálico nos prematuraos com e sem DBP

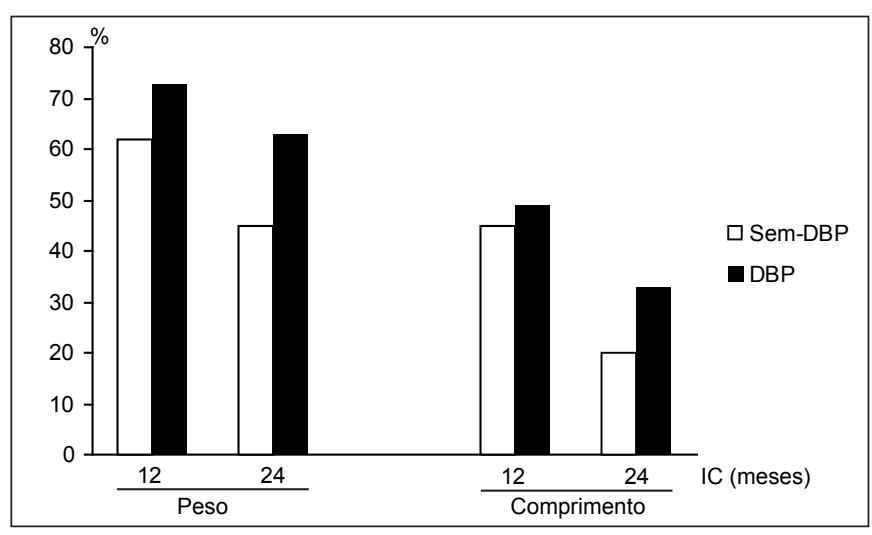

Figura 2 - Freqüencia de falha de crescimento nos prematuros com e sem DBP 
Tabela 2 - Valores médios ( $x \pm D P$ ) e medianos ( $m$ d )dos escores-z de peso, desde o nascimento até 24 meses de idade corrigida, nos prematuros com e sem DBP

\begin{tabular}{lcrrrrrc}
\hline & \multicolumn{3}{c}{ Sem DBP } & & DBP & \\
& no RN & x \pm DP & md & no RN & x \pm DP & md & Valor $\boldsymbol{p}$ \\
\hline Nascimento & 29 & $-3,9 \pm 0,3$ & $-4,0$ & 41 & $-3,8 \pm 0,4$ & $-4,0$ & 0,547 \\
40 semanas & 25 & $-4,6 \pm 0,7$ & $-4,6$ & 30 & $-5,6 \pm 1,1$ & $-5,5$ & $<0,001$ \\
3 meses & 27 & $-1,6 \pm 1,1$ & $-1,6$ & 40 & $-2,4 \pm 1,1$ & $-2,5$ & 0,005 \\
6 meses & 27 & $-1,6 \pm 1,2$ & $-1,6$ & 35 & $-2,4 \pm 1,4$ & $-2,2$ & 0,021 \\
9 meses & 22 & $-1,9 \pm 1,7$ & $-1,8$ & 33 & $-2,3 \pm 1,2$ & $-2,2$ & 0,428 \\
12 meses & 29 & $-2,0 \pm 1,6$ & $-1,9$ & 41 & $-2,6 \pm 1,2$ & $-2,6$ & 0,131 \\
18 meses & 19 & $-2,2 \pm 1,8$ & $-2,0$ & 24 & $-2,4 \pm 1,4$ & $-2,7$ & 0,605 \\
24 meses & 20 & $-1,7 \pm 1,7$ & $-1,5$ & 30 & $-2,4 \pm 1,3$ & $-2,6$ & 0,140 \\
\hline
\end{tabular}

Tabela 3 - Valores médios ( $x \pm D P$ ) e medianos $(\mathrm{md})$ dos escores-z de comprimento, desde o nascimento até 24 meses de idade corrigida, nos prematuros com e sem DBP

\begin{tabular}{|c|c|c|c|c|c|c|c|}
\hline & \multicolumn{3}{|c|}{ Sem DBP } & \multicolumn{3}{|c|}{ DBP } & \multirow[b]{2}{*}{ Valor $p$} \\
\hline & no RN & $x \pm D P$ & $\mathrm{md}$ & no RN & $x \pm D P$ & md & \\
\hline Nascimento & 29 & $-7,8 \pm 1,5$ & $-7,7$ & 41 & $-7,9 \pm 2,5$ & $-8,2$ & 0,962 \\
\hline 40 semanas & 25 & $-6,5 \pm 1,6$ & $-6,8$ & 30 & $-7,9 \pm 2,2$ & $-7,1$ & 0,008 \\
\hline 3 meses & 27 & $-2,4 \pm 1,7$ & $-2,0$ & 40 & $-3,1 \pm 1,4$ & $-3,0$ & 0,080 \\
\hline 6 meses & 27 & $-1,9 \pm 1,4$ & $-1,5$ & 35 & $-2,5 \pm 1,4$ & $-2,3$ & 0,109 \\
\hline 9 meses & 22 & $-1,5 \pm 1,3$ & $-1,4$ & 33 & $-1,7 \pm 1,6$ & $-1,5$ & 0,505 \\
\hline 12 meses & 29 & $-1,3 \pm 1,3$ & $-1,4$ & 41 & $-1,8 \pm 1,0$ & $-1,8$ & 0,153 \\
\hline 18 meses & 19 & $-1,3 \pm 1,1$ & $-1,1$ & 24 & $-1,3 \pm 1,1$ & $-1,4$ & 0,969 \\
\hline 24 meses & 20 & $-1,0 \pm 1,1$ & $-0,9$ & 30 & $-1,5 \pm 1,2$ & $-1,4$ & 0,193 \\
\hline
\end{tabular}

Tabela 4 - Valores médios ( $x \pm D P$ ) e medianos (md) dos escores-z de perímetro cefálico, desde o nascimento até 24 meses de idade corrigida, nos prematuros com e sem DBP

\begin{tabular}{lccccccc}
\hline & \multicolumn{3}{c}{ Sem DBP } & & \multicolumn{2}{c}{ DBP } \\
no RN & x \pm DP & md & no RN & x \pm DP & md & Valor $\boldsymbol{p}$ \\
\hline Nascimento & 29 & $-7,6 \pm 2,5$ & $-8,7$ & 41 & $-7,1 \pm 3,2$ & $-8,1$ & 0,784 \\
4 semanas & 25 & $-5,8 \pm 2,1$ & $-5,2$ & 30 & $-5,9 \pm 2,3$ & $-5,4$ & 0,871 \\
3 meses & 27 & $-1,4 \pm 1,3$ & $-1,2$ & 40 & $-2,2 \pm 1,9$ & -20 & 0,085 \\
6 meses & 27 & $-1,2 \pm 1,2$ & $-1,1$ & 35 & $-2,3 \pm 1,8$ & $-1,7$ & 0,011 \\
9 meses & 22 & $-0,9 \pm 1,5$ & $-0,9$ & 33 & $-1,8 \pm 1,6$ & $-1,6$ & 0,044 \\
12 meses & 28 & $-0,7 \pm 1,3$ & $-0,7$ & 37 & $-1,7 \pm 1,8$ & $-1,3$ & 0,026 \\
18 meses & 19 & $-0,9 \pm 1,6$ & $-0,6$ & 24 & $-1,4 \pm 1,7$ & $-1,3$ & 0,413 \\
24 meses & 20 & $-0,9 \pm 1,3$ & $-0,7$ & 26 & $-1,7 \pm 1,6$ & $-1,4$ & 0,134 \\
\hline
\end{tabular}

Tabela 5 - Presença de catch-up nos prematuros com e sem DBP aos 12 e 24 meses de idade corrigida

\begin{tabular}{lcrrrr}
\hline & $\begin{array}{c}\text { Momentos } \\
\text { de avaliação }\end{array}$ & $\begin{array}{c}\text { Total } \\
\mathbf{n}(\%)\end{array}$ & $\begin{array}{c}\text { Sem DBP } \\
\mathbf{n}(\%)\end{array}$ & $\begin{array}{c}\text { DBP } \\
\mathbf{n}(\%)\end{array}$ & $\begin{array}{c}\text { Sem DBP } \\
\text { versus DBP }\end{array}$ \\
\hline \multirow{2}{*}{ Peso } & 12 meses & $4 / 70(6)$ & $1 / 29(3)$ & $3 / 41(7)$ & 0,492 \\
& 24 meses & $8 / 50(16)$ & $2 / 20(10)$ & $6 / 30(20)$ & 0,345 \\
Comprimento & 12 versus 24 meses & $p<0,001$ & $p=0,042$ & $p=0,011$ & \\
& 12 meses & $14 / 70(20)$ & $4 / 29(14)$ & $10 / 41(24)$ & 0,275 \\
Perímetro & 24 meses & $13 / 50(26)$ & $3 / 20(15)$ & $10 / 30(33)$ & 0,148 \\
Cefálico & 12 versus 24 meses & $p=0,379$ & $p=0,615$ & $p=0,545$ & \\
& 12 meses & $9 / 65(14)$ & $5 / 29(17)$ & $4 / 36(11)$ & 0,477 \\
& 24 meses & $9 / 45(20)$ & $3 / 20(15)$ & $6 / 25(24)$ & 0,453 \\
\hline
\end{tabular}


centual de prematuros que apresentaram falha de crescimento ponderal e linear no final do primeiro e segundo ano de vida. Não houve diferença significante entre os prematuros com e sem DBP, nem entre o primeiro e o segundo ano de vida.

A regressão logística mostrou que, dentre os fatores maternos e gestacionais investigados nesse estudo, nenhum atuou como fator independente de risco para a ocorrência de falha no crescimento ponderal dos prematuros. As variáveis neonatais incluíram idade gestacional, peso e comprimento ao nascer, adequação do peso para a idade gestacional, presença de DBP, uso de corticóide pós-natal e escore-z de peso com 40 semanas. $O$ único fator preditor da falha de crescimento foi o escore-z de peso com 40 semanas. A cada diminuição de um ponto nesse escore, aumentou três vezes a chance de a criança ter falha no crescimento ao final do primeiro ano, com OR $=2,9($ IC95\% $=1,3 ; 6,5)$ e aumentou quatro vezes a chance de falha no segundo ano de vida, com OR=3,9 (IC $95 \%=1,4 ; 11,0)$.

\section{Discussão}

A adesão de $85 \%$ ao seguimento ambulatorial, neste estudo, foi muito satisfatória, considerando-se a baixa condição socioeconômica familiar e a situação de apenas $36 \%$ residirem no município. Entretanto, estes fatos podem ter colaborado para a perda de seguimento, que, ao final do segundo ano, atingiu $28 \%$, inviabilizando a continuidade da pesquisa. Dificuldade em manter a coorte é um dos principais problemas em estudos de seguimento, sendo relatadas taxas de perdas de 20 a $40 \%{ }^{(5,16-18)}$.

A importância da nutrição em etapas precoces da vida e suas repercussões na saúde da criança e do adulto têm sido enfatizadas atualmente, com especial atenção à nutrição do prematuro durante a internação e após a alta ${ }^{(4-6,19,20)}$, bem como a preocupação com o desmame precoce e a escassez do aleitamento materno exclusivo em prematuros ${ }^{(20,21)}$. Este fato é preocupante, pois as fórmulas especiais para prematuros após a alta não são comercializadas em nosso meio e a baixa condição socioeconômica da população é um fator limitante para a aquisição das fórmulas disponíveis no mercado nacional. Assim, pode ocorrer um "choque" nutricional nas primeiras semanas pós-alta, comprometendo a etapa de aceleração máxima do crescimento entre 36 e 40 semanas $^{3-6,19}$. Isto ficou evidente nesse estudo pela piora do escore-z de peso entre o nascimento e 40 semanas, reforçando que a restrição do crescimento pós-natal é um sério problema nos prematuros internados em $\mathrm{UTI}^{(3,2022)}$. Estudo recente com prematuros de
EBP mostrou diminuição dos escores-z de peso, comprimento e perímetro cefálico entre o nascimento e o termo, a qual foi mais acentuada nos adequados para a idade gestacional do que nos pequenos ${ }^{(23)}$.

No presente estudo o catch-down inicial do crescimento foi mais acentuado nos prematuros com displasia, o que pode ser devido à somatória de fatores de risco nutricional antes e após a alta. É classicamente referido que a DBP associa-se com um pior crescimento nos primeiros anos, comprometendo principalmente o peso ${ }^{(7-9,17)}$. Entretanto o padrão de crescimento e a época do catch-up ainda não estão bem definidos nas crianças displásicas.

É discutível qual a melhor maneira de avaliar o crescimento de prematuros. No presente estudo, optou-se pela avaliação dos escores-z nas curvas de crescimento do CDCNCHS 2000, que são adotadas como referência no Serviço e também as mais utilizadas nos estudos sobre crescimento ${ }^{(12)}$. Entretanto, tais curvas apresentam o padrão de normalidade do crescimento de crianças americanas e não são específicas para prematuros; por isso, os escores-z de nossos prematuros devem ter sido substancialmente baixos ao nascimento.

A evolução dos escores-z neste estudo confirmou nossa hipótese que os prematuros de EBP apresentam catch-up do crescimento nos primeiros anos de vida e que a DBP interfere no catch-up.

Dos três parâmetros antropométricos avaliados, o perímetro cefálico mostrou melhor perfil de crescimento, com catch-up precoce nos dois grupos, atingindo a faixa de normalidade já no primeiro ano. Esse é um resultado importante, pois o inadequado crescimento do perímetro cefálico nos primeiros anos está associado ao pior prognóstico de desenvolvimento ${ }^{(24)}$. A expectativa de crescimento do perímetro cefálico é boa para prematuros de muito baixo peso ${ }^{(25)}$, mas para prematuros menores que $1.000 \mathrm{~g}$, os resultados não são animadores $^{(9)}$.

O crescimento linear também foi bastante satisfatório em nossos prematuros, principalmente no grupo sem DBP, que apresentou catch-up precoce, atingindo valores entre -2 e zero a partir do segundo trimestre. Mesmo os displásicos, com catch-up um pouco mais tardio, atingiram a faixa de normalidade. Resultados semelhantes foram obtidos em prematuros displásicos graves que tiveram alta em uso de oxigênio e, no seguimento, $88 \%$ apresentaram $c a t c h-u p$ do comprimento nos primeiros dois anos ${ }^{(26)}$.

Em nosso estudo, o peso teve a pior evolução, especialmente nos prematuros com DBP que, apesar do catch-up no primeiro trimestre, não conseguiram atingir a faixa de nor- 
malidade até o final do segundoano. A elevada morbidade e a necessidade de reinternação podem ter contribuído para essa evolução. O'Callaghan et al detectaram 50\% de reinternações e crescimento ponderal inadequado em prematuros de EBP, nos primeiros dois anos de vida ${ }^{(18)}$.

As diferenças no crescimento de nossos prematuros com e sem displasia predominaram no primeiro semestre de vida, e o catch-up foi mais irregular e atrasado nos displásicos. Resultados semelhantes foram obtidos por Huysman et al em prematuros extremos displásicos, os quais evoluíram com baixos escores-z de peso e comprimento em todas as avaliações no primeiro ano de vida, sendo a evolução do peso pior que do que a do comprimento ${ }^{(8)}$.

Em estudo multicêntrico, 406 prematuros de muito baixo peso foram avaliados na idade escolar. O peso e o perímetro cefálico foram menores nas crianças que tiveram DBP, mas ao controlar os potenciais fatores de confusão, as diferenças desapareceram, sugerindo que o crescimento inadequado pode estar associado a vários outros fatores e não à displasia ${ }^{(17)}$.

Apesar da evolução favorável no crescimento de nossos prematuros, o catch-up realizado não foi suficiente para equiparálos aos recém-nascidos de termo sadios e elevado percentual de crianças não atingiu o percentil 5 das curvas do CDC-NCHS no segundo ano de vida: $56 \%$ para peso, $28 \%$ para comprimento e $32 \%$ para perímetro cefálico. Na literatura, o percentual de crescimento inadequado é variável, com valores de 17-43\% para peso, $10-17 \%$ para comprimento e $5-38 \%$ para perímetro cefálico, nos primeiros três anos de vida ${ }^{(9,18,27)}$. Tais diferenças podem ser devidas a variações no padrão genético e alimentar, diferenças na qualidade de vida das crianças conforme o grau de desenvolvimento do país, bem como heterogeneidade das curvas de referência adotadas.

A análise multivariada mostrou que a DBP não teve influência direta na ocorrência de falha de crescimento em nossos prematuros, enquanto o escore-z de peso com 40 semanas foi a variável preditora do peso aos 12 e 24 meses idade corrigida. Este resultado é concordante com dois importantes estudos sobre falha de crescimento em prematuros. Kelleher et al detectou 19,7\% de falha de crescimento nos primeiros três anos de vida, sendo que mais de $80 \%$ dos casos de falha não foram decorrentes de doenças crônicas como a DBP, mas sim da interação de fatores adversos biológicos e ambientais ${ }^{(28)}$. Dusick et al estudaram 1.151 prematuros menores que $1.000 \mathrm{~g}$ e documentaram $89 \%$ de falha de crescimento com 36 semanas de idade corrigida, que se associou com elevada taxa de falha de crescimento aos dois anos (40\%). A DBP e o uso prolongado de oxigênio não foram preditores de falha de crescimento ${ }^{(5)}$.
A DBP, definida pela necessidade de oxigênio aos 28 dias, pode ter atuado de forma indireta, traduzindo maior gravidade dos prematuros, nos quais a baixa condição socioeconômica familiar aliada à elevada morbidade tenham culminado na inadequada condição nutricional no início da vida, representada pelo catch-down do peso entre o nascimento e o termo, que foi o fator determinante para a ocorrência de falha de crescimento.

As taxas de falha de crescimento em nossa casuística devem ser interpretadas com cautela, pois uma característica do estudo foi restringir o período de avaliação até o final do segundo ano de vida. Ao analisar o percentual de crianças que estavam realizando $c a t c h-u p$ no final de cada ano, detecta-se catch-up de comprimento em cerca de $1 / 4$ da amostra aos 24 meses de idade corrigida e aumento significante no percentual de catch-up do peso entre 12 e 24 meses. Isto traduz o potencial de recuperação mais tardio do crescimento nesses prematuros de EBP, com e sem DBP, o que tem sido destacado nos estudos de seguimento em longo prazo ${ }^{(29,30)}$.

Esta investigação tem algumas limitações: foi unicêntrica; não foi avaliada a estatura dos pais, o que impossibilitou investigar o efeito do potencial genético no crescimento linear; o período de recrutamento da coorte foi longo, com possibilidade de alterações nas práticas nutricionais durante a internação; também não foi avaliado o efeito do tempo de oxigenoterapia no crescimento dos prematuros displásicos.

Apesar das limitações, este é, a nosso conhecimento, o primeiro estudo nacional sobre seguimento de prematuros menores que $1.000 \mathrm{~g}$ por um período de dois anos, envolvendo casuística relativamente ampla, cujas características refletem o perfil da clientela atendida na maioria das unidades neonatais em nosso meio. Os resultados obtidos podem colaborar para o maior conhecimento do prognóstico de crescimento desses prematuros, balizar as expectativas médicas e familiares, alertar para a importância da desnutrição pós-natal nos prematuros $<1.000 \mathrm{~g}$ e estimular os colegas a realizar o seguimento de recém-nascidos prematuros, em longo prazo.

Pode-se, portanto, concluir que prematuros de EBP apresentam crescimento satisfatório, mas muitos não atingem a faixa de normalidade na curva de referência até o final do segundo ano. Os primeiros três meses após o termo representam o período crítico para a ocorrência de catch-up, cuja seqüência é: perímetro cefálico, comprimento e peso. Prematuros com DBP apresentam atraso no catch-up, pior crescimento ponderal e alta taxa de falha de crescimento nos primeiros dois anos de vida. $\mathrm{O}$ catch-down do peso entre o nascimento e 40 semanas é fator de risco para falha de crescimento nos primeiros dois anos de vida. 


\section{Referências bibliográficas}

1. Hack M, Fanaroff AA. Outcomes of children of extremely low birth weight and gestational age in the 1990s. Semin Neonatol 2000;5:89-106.

2. Rugolo LM. Crescimento e desenvolvimento a longo prazo do prematuro extremo. J Pediatr (Rio J) 2005;81:S101-10.

3. Ehrenkranz RA. Growth outcomes of very low-birth weight infants in the newborn intensive care unit. Clin Perinatol 2000;27:325-45.

4. Heird WC. Determination of nutritional requirements in preterm infants, with special reference to "catch-up" growth. Semin Neonatol 2001;6:365-75.

5. Dusick AM, Poindexter BB, Ehrenkranz RA, Lemons JA. Growth failure in the preterm infant: can we catch-up? Semin Perinatol 2003;27:302-10

6. Camelo Jr JS, Martinez FE. Dilemas nutricionais no pré-termo extremo e repercussões na infância, adolescência e vida adulta. J Pediatr (Rio J) 2005;81:S33-42.

7. Vohr BR, Bell EF, Oh W. Infants with bronchopulmonary dysplasia: growth pattern and neurologic developmental outcome. Am J Dis Child 1982;136: 443-7.

8. Huysman WA, de Ridder M, de Bruin NC, van Helmond G, Terpstra N, van Goudoever JB et al. Growth and body composition in preterm infants with bronchopulmonary dysplasia. Arch Dis Child Fetal Neonatal Ed 2003;88: F46-F51

9. Wood NS, Costeloe K, Gibson AT, Hennessy EM, Marlow N, Wikinson AR et al. The EPICure study: growth and associated problems in children born at 25 weeks of gestational age or less. Arch Dis Child Fetal Neonatal Ed 2003;88:F492-500.

10. Bancalari E, Abdenour GE, Feller R, Gannon J. Bronchopulmonary dysplasia: clinical presentation. J Pediatr 1979;95:819-23.

11. Ong KK, Ahmed ML, Emmett PM, Preece MA, Dunger DB. Association between postnatal catch-up growth and obesity in childhood: prospective cohort study. BMJ 2000;320:967-71.

12. Kuczmarski RJ, Ogden CL, Grummer-Strawn LM, Flegal KM, Guo SS, Wei R et al. CDC growth charts: United States. Adv Data 2000;314:1-27.

13. Gouveia AJ. Professores de amanhã: um estudo de escolha profissional. $2^{\text {a }}$ ed. São Paulo: Livraria Pioneira; 1970.

14. Alexander GR, Himes JH, Kaufman RB, Mor J, Kogan M. A United States national reference for fetal growth. Obstet Gynecol 1996;87:163-8.

15. Papile LA, Burstein J, Burstein R, Koffler H. Incidence and evolution of subependymal and intraventricular hemorrhage: a study of infants with birth weights less than 1,500 gm. J Pediatr 1978;92:529-34.

16. Saigal S. Follow-up of very low birthweight babies to adolescence. Semin Neonatol 2000;5:107-18.
17. Vrlenich LA, Bozynski ME, Shyr Y, Schork A, Roloff DW, McCormick MC. The effect of bronchopulmonary dysplasia on growth at school age. Pediatrics 1995;95:855-9.

18. O'Callaghan MJ, Burns Y, Gray P, Harvey JM, Mohay HI, Rogers $\mathrm{Y}$ et al. Extremely low birth weight and control infants at 2 years corrected age: a comparison of intellectual abilities, motor performance, growth and health. Early Hum Dev 1995;40:115-28.

19. Fewtrell MS. Growth and nutrition after discharge. Semin Neonato 2003;8:169-76.

20. Anchieta LM, Xavier CC, Colosimo EA. Crescimento de recém-nascidos prétermo nas primeiras 12 semanas de vida. J Pediatr (Rio J) 2004;80:267-76.

21. Furman L, Minich NM, Hack M. Breastfeeding of very low birth weight infants. J Hum Lact 1998;14:29-34.

22. Cooke RJ, Ainsworth SB, Fenton AC. Postnatal growth retardation: a universal problem in preterm infants. Arch Dis Child Fetal Neonatal Ed 2004;89:F428-30.

23. Jordan IM, Francart J, Sann L, Putet G. Growth in extremely low birth weight infants up to three years. Biol Neonate 2005;88:57-65.

24. Peterson J, Taylor HG, Minich N, Klein N, Hack M. Subnormal head circumference in very low birth weight children: neonatal correlates and school-age consequences. Early Hum Dev 2006;82:325-34.

25. Brandt I, Sticker EJ, Lentze MJ. Catch-up growth of head circumference of very low birth weight, small for gestational age preterm infants and mental development to adulthood. J Pediatr 2003;142:463-8.

26. Wach R, O'Hare JL, Primhak S, Primhak RA. Patterns of statural growth in infants with chronic lung disease of prematurity (CLD). Thorax 2000;55:A57.

27. Finnstrom O, Otterblad Olausson PO, Sedin G, Serenius F, Svenningsen N, Thiringer $\mathrm{K}$ et al. Neurosensory outcome and growth at three years in extremely low birthweight infants: follow-up results from the Swedish national prospective study. Acta Paediatr 1998;87:1055-60.

28. Kelleher KJ, Casey PH, Bradley RH, Pope SK, Whiteside L, Barrett KW et al. Risk factors and outcomes for failure to thrive in low birth weight preterm infants. Pediatrics 1993;91:941-8.

29. Qvigstad E, Verloove-Vanhorick SP, Ens-Dokkum MH, Schreuder AM, Veen S, Brand $R$ et al. Prediction of height achievement at five years of age in children born very preterm or with very low birth weight: continuation of catch-up growth after two years of age. Acta Paediatr 1993;82:444-8.

30. Doyle LW, Faber B, Callanan C, Ford GW, Davis NM. Extremely low birth weight and body size in early adulthood. Arch Dis Child 2004;89:347-50. 